\title{
Measurement of light charged particles in the decay channels of medium-mass excited compound nuclei
}

\author{
S. Valdré ${ }^{1,2, a}$, S. Barlini ${ }^{1}$, G. Casini ${ }^{2}$, G. Pasquali ${ }^{1,2}$, S. Piantelli ${ }^{2}$, S. Carboni ${ }^{1,2}$, M. Cinausero ${ }^{3}$, F. $^{2}$ \\ Gramegna $^{3}$, T. Marchi ${ }^{3,4}$, G. Baiocco ${ }^{5}$, L. Bardelli ${ }^{2}$, G. Benzoni ${ }^{6}$, M. Bini ${ }^{1,2}$, N. Blasi ${ }^{6}$, A. Bracco ${ }^{6,7}$, \\ S. Brambilla ${ }^{6}$, M. Bruno ${ }^{5}$, F. Camera ${ }^{6,7}$, A. Corsi ${ }^{6,7}$, F. Crespi ${ }^{6,7}$, M. D'Agostino ${ }^{5}$, M. Degerlier ${ }^{3}$, \\ V. L. Kravchuk ${ }^{3}$, S. Leoni ${ }^{6,7}$, B. Million ${ }^{6,7}$, D. Montanari ${ }^{4}$, L. Morelli ${ }^{5}$, A. Nannini ${ }^{2}$, R. Nicolini ${ }^{6,7}$, \\ G. Poggi ${ }^{1,2}$, G. Vannini ${ }^{5}$, O. Wieland ${ }^{6}$, P. Bednarczyk ${ }^{8}$, M. Ciemała ${ }^{8}$, J. Dudek ${ }^{9}$, B. Fornal ${ }^{8}$, M. \\ $\mathrm{Kmiecik}^{8}$, A. Maj ${ }^{8}$, M. Matejska-Minda ${ }^{8}$, K. Mazurek ${ }^{8}$, W. Męczyński ${ }^{8}$, S. Myalski ${ }^{8}$, J. Styczeń ${ }^{8}$, \\ and M. Ziębliński ${ }^{8}$
}

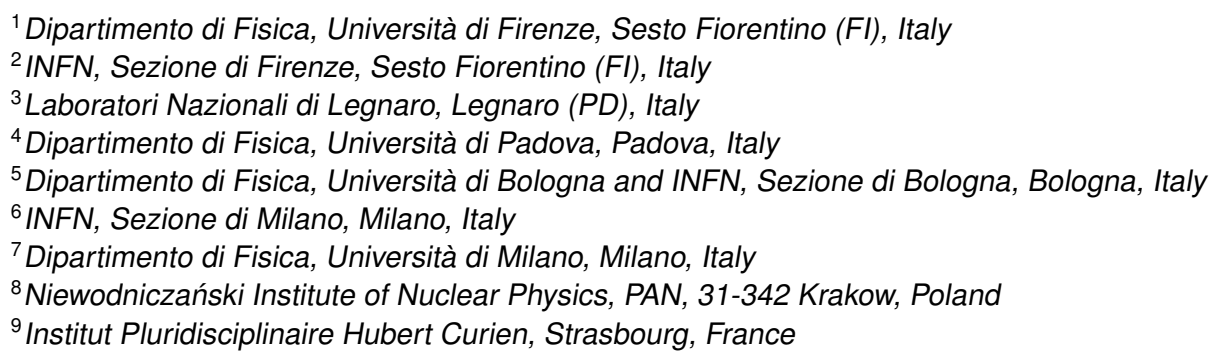

\begin{abstract}
The ${ }^{48} \mathrm{Ti}$ on ${ }^{40} \mathrm{Ca}$ reactions have been studied at 300 and $600 \mathrm{MeV}$ focusing on the fusion-evaporation (FE) and fusion-fission (FF) exit channels. Energy spectra and multiplicities of the emitted light charged particles have been compared to Monte Carlo simulations based on the statistical model.

Indeed, in this mass region $(A \sim 100)$ models predict that shape transitions can occur at high spin values and relatively scarce data exist in the literature about coincidence measurements between evaporation residues and light charged particles. Signals of shape transitions can be found in the variations of the lineshape of high energy gamma rays emitted from the de-excitation of GDR states gated on different region of angular momenta. For this purpose it is important to keep under control the FE and FF processes, to regulate the statistical model parameters and to control the onset of possible preequilibrium emissions from 300 to $600 \mathrm{MeV}$ bombarding energy.
\end{abstract}

\section{Introduction}

Macroscopic nuclear models [1] predict a rapid transition from oblate to prolate shape at high spin for medium-mass nuclei; this transition can be observed thanks to the low-fissility of these systems which can therefore sustain high spins with limited fission probability. Variations of the GDR spectra can signal this process but, since the effects are small, it is important an accurate description of the

\footnotetext{
ae-mail: valdre@fi.infn.it
}

This is an Open Access article distributed under the terms of the Creative Commons Attribution License 2.0, which permits unrestricted use, distribution, and reproduction in any medium, provided the original work is properly cited. 

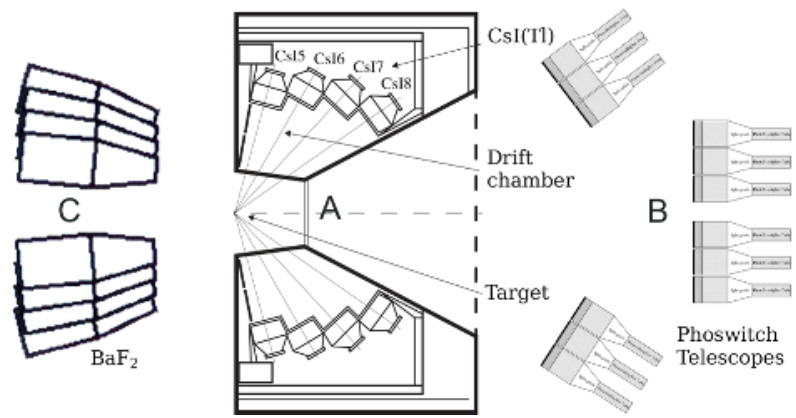

Figure 1. The experimental setup was composed of the GARFIELD apparatus (A), a wall of Phoswich detectors (B) and the HECTOR $\mathrm{BaF}_{2}$ crystals (C).

decay of the excited compound nuclei $(\mathrm{CN})$. With this in mind and considering that not many data in this region exist from exclusive measurements [2-4], we performed an experimental campaign at the Tandem-ALPI complex of the Laboratori Nazionali di Legnaro. ${ }^{88}$ Mo compoud nuclei have been studied, formed in the reactions of ${ }^{48} \mathrm{Ti}$ on ${ }^{40} \mathrm{Ca}$ target $\left(500 \mu \mathrm{g} / \mathrm{cm}^{2}\right)$ at 300 and $600 \mathrm{MeV}$ bombarding energies. Table 1 reports some parameters for these reactions.

Table 1. Compound nucleus excitation energy, vanishing fission barrier spin and grazing angular momentum and estimated total reaction cross section reported for both bombarding energies.

\begin{tabular}{ccccc}
\hline $\boldsymbol{E}[\mathbf{M e V}]$ & $\boldsymbol{\varepsilon}^{*}[\mathbf{M e V} / \mathbf{u}]$ & $\boldsymbol{l}_{\mathbf{0}}\left(B_{f}=0\right)[\hbar]$ & $\boldsymbol{l}_{\boldsymbol{g r}}[\hbar]$ & $\boldsymbol{\sigma}_{\boldsymbol{R}}[\mathbf{b a r n}]$ \\
\hline 300 & 1.4 & 64 & 88.4 & 1.7 \\
600 & 3.0 & 64 & 149.2 & 2.5 \\
\hline
\end{tabular}

The reactions have been analyzed focusing on the fusion-evaporation (FE) and fusion-fission (FF) exit channels, which dominate in mass-symmetric collisions at moderate bombarding energies. For excitation energies up to around $3 \mathrm{MeV} / \mathrm{u}$ the equilibrium of the internal degrees of freedom is reached in shorter times compared to evaporation and fission time scales. Thus it is reasonable to adopt, as a first approximation, the statistical model of compound nucleus to study its decay. Experimental data are compared with events produced through Monte Carlo simulations (performed with the well known Gemini++ code [4]) where complete fusion events are generated with a triangular spin distribution for the $\mathrm{CN}$ up to $l_{0}$. The output of the simulation is then filtered through solid angle acceptance and particle energy detection thresholds of the experimental apparatus.

\section{Experimental apparatus}

The experimental setup is shown in Figure 1. Phoswich telescopes [5] (made of two plastic scintillators and a $\mathrm{CsI}(\mathrm{Tl})$ crystal) were used to select the exit channel (FE/FF) by detecting heavy residues or fragments in the polar range from $6^{\circ}$ to $12^{\circ}$, also allowing charge identification up to about $Z \sim 12$. The forward chamber of the GARFIELD apparatus $[6,7]$ was the main detector for the LCP emitted in the reaction. It consists of $96 \Delta E-E$ telescopes where the $\Delta E$ stage is a gas detector with moderate multiplication, followed by a $\mathrm{CsI}(\mathrm{Tl})$ crystal $4 \mathrm{~cm}$ thick. The 96 telescopes are organized in four rings (referred as $8,7,6,5$ ), from $29^{\circ}$ to $85^{\circ}$ (polar range) and 24 azimuthal sectors, thus covering a big 
part of the forward emisphere $(\sim 1.5 \pi \mathrm{sr})$. At backward angles $\left(>90^{\circ}\right)$ the HECTOR array made of eight big $\mathrm{BaF}_{2}$ crystals was used to measure hard gamma rays associated to GDR.

The $E$ vs tof technique was used to detect the heavy fragments (and hence also ER) in the Phoswich detectors. Via $\Delta E-E$ correlations the Phoswich also allowed charge identification for intermediate mass fragments (IMF) and mass determination for light charged particles (LCP).
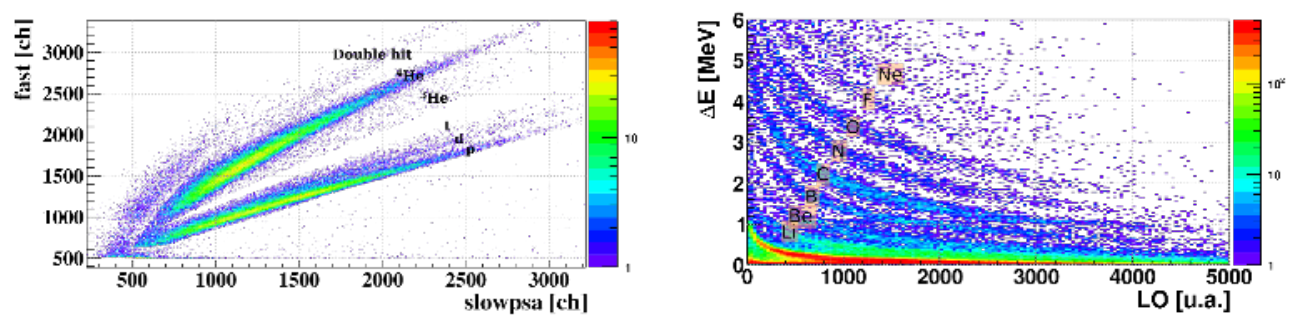

Figure 2. Particle identification in GARFIELD. Isotopes of light charged particles are discriminated via fast slow technique (left). Instead $\Delta E-E$ (right) is used for IMF charge identification.

LCP in GARFIELD are identified through the usual fast - slow correlation in CsI(Tl) crystals as shown in Figure 2 (left panel). Heavier fragments are identified with the $\Delta E-E$ method using the gas stage (right panel of Figure 2).

\section{Data analysis}

To obtain " $4 \pi$ " particle multiplicities we used simulated data to correct for the efficiency of the apparatus. In particular, for each particle species, the multiplicity measured in a given set of detectors has been divided by the efficiency of the same set of detectors, evaluated by the simulations. The LCP multiplicities (obtained from Phoswich data only) in coincidence with an ER are shown in Table 2 for the two measured energies and they are compared to statistical model predictions.

Table 2. Number of LCP emitted per ER. Yields are obtained from Phoswich detectors only and they are corrected for the efficiency. Not enough statistics has been measured for tritons which are not reported.

\begin{tabular}{ccccccc}
\hline & \multicolumn{3}{c}{$\mathbf{3 0 0} \mathbf{~ M e V}$} & \multicolumn{3}{c}{$\mathbf{6 0 0 ~ M e V}$} \\
& $\mathbf{m}_{\mathbf{p}}$ & $\mathbf{m}_{\mathbf{d}}$ & $\mathbf{m}_{\alpha}$ & $\mathbf{m}_{\mathbf{p}}$ & $\mathbf{m}_{\mathbf{d}}$ & $\mathbf{m}_{\alpha}$ \\
\hline EXP & $4.0 \pm 0.8$ & $0.18 \pm 0.07$ & $2.9 \pm 0.6$ & $5.5 \pm 1.1$ & $0.7 \pm 0.3$ & $3.8 \pm 0.8$ \\
MC & 3.5 & 0.08 & 1.1 & 5.9 & 0.53 & 1.8 \\
\hline
\end{tabular}

As expected, the measured (and predicted) multiplicities at $600 \mathrm{MeV}$ are larger than at $300 \mathrm{MeV}$. Protons appear to be reasonably reproduced by the statistical model, run with default values of input parameters [4]. Instead, at both energies data show an excess of $\alpha$ particles with respect to Gemini++ predictions. At $300 \mathrm{MeV}$ also deutons are more abudantly emitted with respect to calculations. The evaluation of LCP multiplicities also using GARFIELD data is in progress.

The $\mathrm{p} / \mathrm{d}$ and $\mathrm{p} / \alpha$ ratios obtained from the Phoswiches and GARFIELD are separately reported in Table 3. From Table 2 and Table 3 one can conclude that more $\alpha$-particles are emitted than predicted by Gemini++ run with standard parameters, and that the excess increases at forward angles (Phoswich region). 
Table 3. LCP yield ratios from GARFIELD and Phoswich data in the FE channel.

\begin{tabular}{ccccc}
\hline & \multicolumn{2}{c}{$300 \mathrm{MeV}$} & \multicolumn{2}{c}{$\mathbf{6 0 0} \mathbf{M e V}$} \\
& $\mathbf{p} / \mathbf{d}$ & $\mathbf{p} / \boldsymbol{\alpha}$ & $\mathbf{p} / \mathbf{d}$ & $\mathbf{p} / \boldsymbol{\alpha}$ \\
\hline EXP from GARFIELD & $29 \pm 12$ & $2.9 \pm 0.6$ & $9.4 \pm 3.8$ & $2.4 \pm 0.5$ \\
EXP from Phoswich & $22 \pm 9$ & $1.4 \pm 0.3$ & $7.8 \pm 3.1$ & $1.4 \pm 0.3$ \\
MC & 42.3 & 3.30 & 11.3 & 3.39 \\
\hline
\end{tabular}
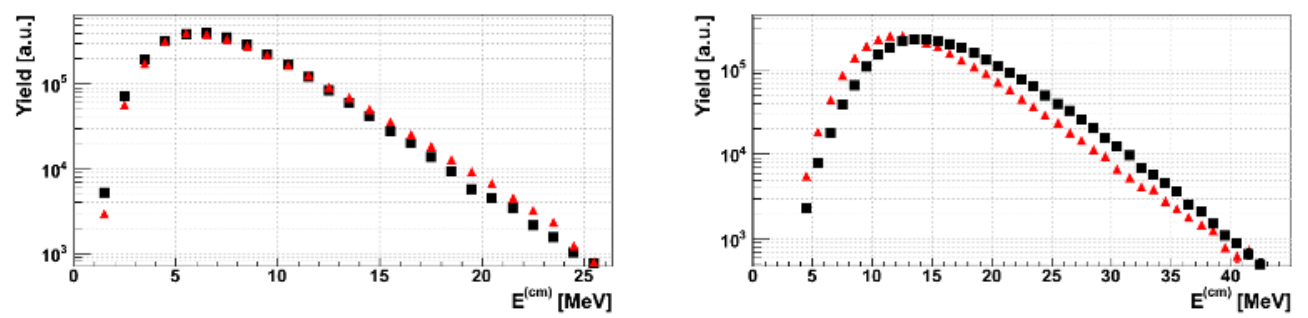

Figure 3. Center of mass energy spectra of LCP detected in GARFIELD between $\theta=29.5^{\circ}$ and $\theta=40.0^{\circ}$ (ring 8 ) in the fusion-evaporation channel at $300 \mathrm{MeV}$. Proton spectrum is on the left while $\alpha$-particle spectrum is on the right. Black squares are experimental points, while red triangles are simulated data. The yields are normalized to the integral for each panel.

The shape of energy spectra (Figure 3) seems to confirm the pure statistical nature of proton emission while some disagreement is found for $\alpha$ particles. In particular, $\alpha$ particles appear to be on average more energetic than predicted and this happens also for the FF channel.

\section{Conclusions and remarks}

The decay of ${ }^{88} \mathrm{Mo}$ at 1.4 and $3.0 \mathrm{MeV} / \mathrm{u}$ excitation energy is under study. Proton emission in the FE channel is well reproduced by the Gemini++ statistical code run with standard input values. An excess of $\alpha$ particles has been found at both bombarding energies also associated to a slightly different $\mathrm{CM}$-energy spectrum with respect to the predicted one. Work is in progress to better quantify this excess and to understand if it could be accounted for by suitable variations of model parameters, in particular to better describe the shape deformation at high spin as pointed out in [8], or it comes for other sources. It is also in progress the study of the fission channel, whose description is important for shape transitions as it is associated to the highest spins where such shape changes are expected.

\section{References}

[1] K. Mazurek et al, Acta Phys. Pol. B 42, 471 (2011)

[2] S.J. Sanders, Phys. Rev. C 44, 2676 (1991)

[3] W. von Oertzen et al., Phys. Rev. C 78, 044615 (2008)

[4] R.J. Charity, Phys. Rev. C 82, 014610 (2010)

[5] M. Bini et al., Nucl. Instr. and Meth. A 515, 497 (2003)

[6] F. Gramegna et al., Nucl. Instr. and Meth. A 389, 474 (1997)

[7] M. Bruno et al., submitted to EPJ A (2013)

[8] B. Fornal et al., Phys. Rev. C 41, 127 (1990) 